Supporting Informations for

\title{
Bridged Polycatenane
}

Nobuhiro Watanabe, Yoshihiro Ikari, Nobuhiro Kihara,* and Toshikazu Takata*

${ }^{1}$ Department of Applied Chemistry, Graduate School of Engineering, Osaka Prefecture University, Gakuen-cho 1-1, Sakai, Osaka 599-8531, Japan

${ }^{2}$ Department of Organic and Polymeric Materials, Tokyo Institute of Technology, $O$ okayama, Meguro-ku, Tokyo 152-8552, Japan

e-mail: kihara@chem.osakafu-u.ac.jp

\section{Synthesis of monomer [2]catenane 4.}

To a solution of macrocycle 1 (145 mg, $0.20 \mathrm{mmol})$ and triethylamine (240 mg, $2.4 \mathrm{mmol})$ in chloroform ( $80 \mathrm{ml}$, stabilized with amylene) were added a solution of 2 (513 $\mathrm{mg}, 1.2 \mathrm{mmol}$ ) in chloform (30 ml) and a solution of $\mathbf{3}(358 \mathrm{mg}, 1.2 \mathrm{mmol})$ in THF (30 ml) simultaneously using a syringe pump over a period of $3 \mathrm{~h}$ at room temperature. After the mixture was stirred for $6 \mathrm{~h}$, white precipitate (mainly ring A) was removed by filtration. The filtrate was washed with $1 \mathrm{M} \mathrm{HCl}$ aq, $5 \% \mathrm{~K}_{2} \mathrm{CO}_{3}$ aq, then water, dried over $\mathrm{MgSO}_{4}$, and evaporated in vacuo. The residue was chromatographed over silica gel using $\mathrm{CHCl}_{3} / \mathrm{MeOH}(100 / 2)$ as an eluent to afford [2]catenane 4 (124 mg, 45\% based on 1) as a white solid. 4 decomposed upon heating without melting to afford polymer $\mathbf{6}$ directly.

${ }^{1} \mathrm{H}$ NMR (400 MHz, $\left.\mathrm{CDCl}_{3}, 323 \mathrm{~K}\right): \delta 8.81$ (s, 1H), 8.73 (br s, 2H), 8.66 (s, 2H), 8.65 (s, 2H), $7.36(\mathrm{~s}, 2 \mathrm{H}), 7.36-7.26(\mathrm{~m}, 6 \mathrm{H}), 7.20(\mathrm{~s}, 2 \mathrm{H}), 7.13(\mathrm{~s}, 2 \mathrm{H}), 7.03(\mathrm{~d}, J=7.6 \mathrm{~Hz}, 2 \mathrm{H}), 7.02$ $(\mathrm{d}, J=7.6 \mathrm{~Hz}, 2 \mathrm{H}), 6.79(\mathrm{~d}, J=8.4 \mathrm{~Hz}, 4 \mathrm{H}), 6.40(\mathrm{~d}, J=8.4 \mathrm{~Hz}, 4 \mathrm{H}), 4.40(\mathrm{~d}, J=4.0 \mathrm{~Hz}$, 4H), 4.09 (t, J=4.8 Hz, 4H), $4.08(\mathrm{~s}, 4 \mathrm{H}), 3.98(\mathrm{~s}, 4 \mathrm{H}), 3.80(\mathrm{t}, J=4.8 \mathrm{~Hz}, 4 \mathrm{H}), 3.67(\mathrm{~s}, 4 \mathrm{H})$, 3.22 (s, 4H), 1.34 (s, 9H) ppm. ${ }^{13} \mathrm{C}$ NMR (100 MHz, $\left.\mathrm{CDCl}_{3}, 323 \mathrm{~K}\right): \delta 170.56,166.76$, $165.61,162.83,158.70,153.07,150.67,149.38,139.84,135.25,134.90,134.44,133.39$, $130.67,129.95,129.77,129.46,129.11,121.21,120.93,120.60,118.71,117.64,116.85$, 
113.13, 112.85, 71.04, 69.86, 67.39, 58.37, 44.72, 35.17, 31.16, 30.40, 29.69 ppm. IR (KBr): $=3352,1740,1640,1602,1529,1252,1139,749 \mathrm{~cm}^{-1}$. MS (FAB, $\left.m \mathrm{NBA}\right): \mathrm{m} / z(\%): 1374.8$ (72) $\left[\mathrm{M}^{+}+\mathrm{H}\right], 653.3(100)$ [ring $\left.\mathrm{A}^{+}+\mathrm{H}\right]$. Anal. Calcd for $\mathrm{C}_{72} \mathrm{H}_{70} \mathrm{~N}_{4} \mathrm{O}_{18} \mathrm{~S}_{3} \cdot \mathrm{CHCl}_{3}, \mathrm{C}, 58.65 ; \mathrm{H}$, 4.79; N, 3.75. Found, C, 58.78; H, 4.88; N, 3.78.

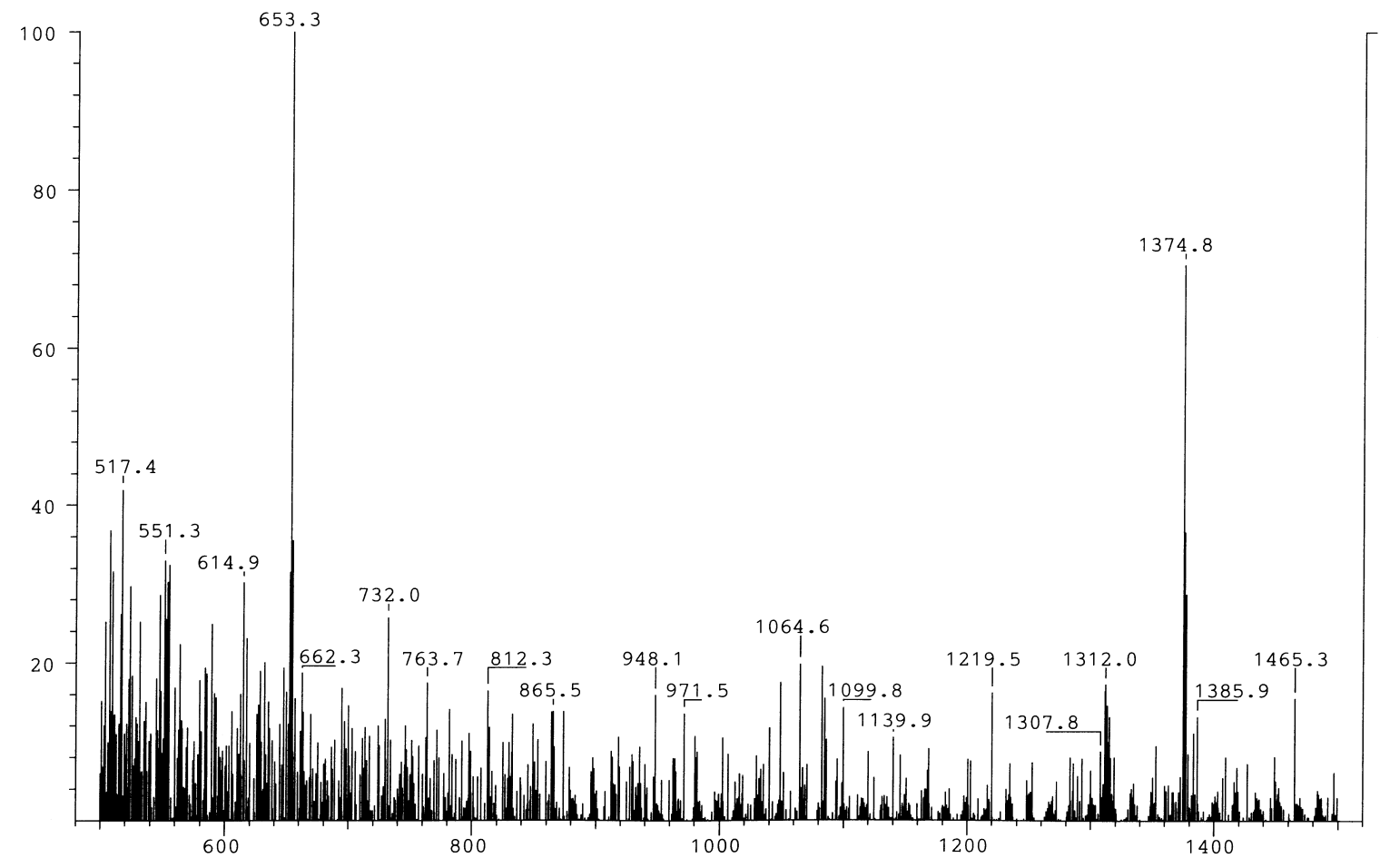

Figure S1. FAB-MS of 4. 


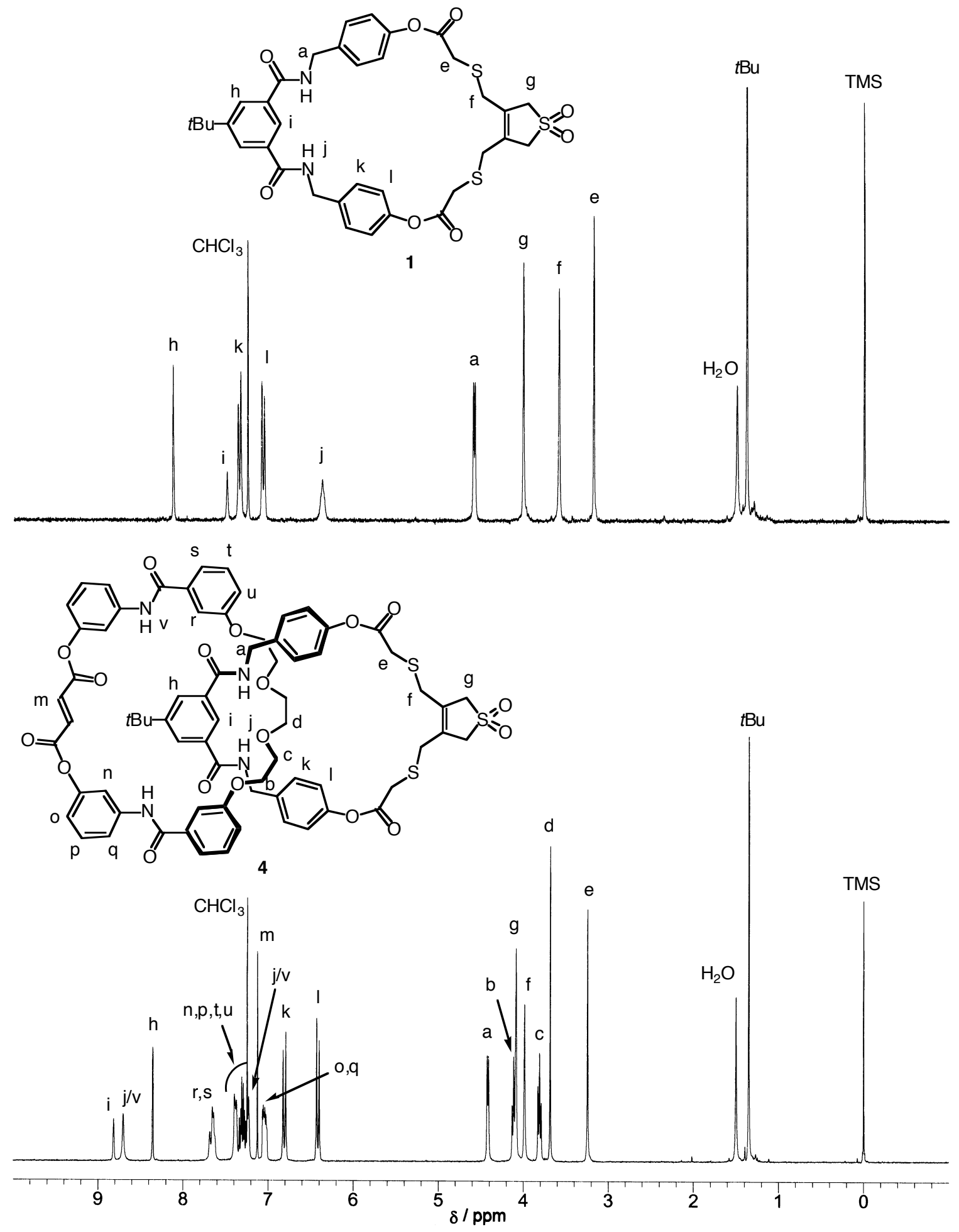

Figure S2. ${ }^{1} \mathrm{H}$ NMR spectra of $\mathbf{1}$ (upper) and $\mathbf{4}$ (lower). 


\section{Polymerization}

A powder of 4 or a solution of 4 in 1,2-dichlorobenzene was heated at $140{ }^{\circ} \mathrm{C}$ for $24 \mathrm{~h}$. In the case of solution polymerization, the solvent was removed after the polymerization by the evaporation in vacuo. The product was analyzed as obtained without further purification.

\section{Dimer 7}

A powder of $4(37 \mathrm{mg}, 26.9 \mu \mathrm{mol})$ was heated at $140{ }^{\circ} \mathrm{C}$ for $5 \mathrm{~min}$. The highest molecular weight fraction was isolated from the reaction mixture by preparative GPC (chloroform), and 4 was recovered. This procedure was repeated five times, and $6.3 \mathrm{mg}(5 \%)$ of the dimer fraction was collected. This product showed a single peak by GPC (chloroform).

${ }^{1} \mathrm{H}$ NMR $\left(270 \mathrm{MHz}, \mathrm{CDCl}_{3}, 323 \mathrm{~K}\right): \delta 8.98-8.69$ (m, 4H), 8.60-8.30 (m, 8H), 7.80-7.58 (m, 6H), 7.57-7.32 (m, 6H), 7.18-7.10 (m, 4H), 7.08-6.91 (m, 12H), 6.85-6.55 (m, 14H), 6.47$6.22(\mathrm{~m}, 10 \mathrm{H}), 4.49-4.33(\mathrm{~m}, 6 \mathrm{H}), 4.27-3.97(\mathrm{~m}, 16 \mathrm{H}), 3.96-3.59(\mathrm{~m}, 28 \mathrm{H}), 3.39-3.28(\mathrm{~m}$, 2H), 3.14-2.53 (m, 6H), 1.42 (s, 9H), 1.37 (s, 9H) ppm. IR (NaCl): 3353, 2960, 2923, 2862, $1742,1645,1602,1540,1267,1137,749 \mathrm{~cm}^{-1}$. MS (FAB, $\left.m \mathrm{NBA}\right): m / z(\%): 2684.0(35)$ $\left[\mathrm{M}^{+}\right]$

\section{Model Compound $\mathbf{8}$}

A solution of $32.4 \mathrm{mg}(21.8 \mu \mathrm{mol})$ of [2]catenane $4,314 \mathrm{mg}(2.18 \mathrm{mmol})$ of dimethyl fumarate, and $1 \mathrm{mg}$ of hydroquinone in $0.5 \mathrm{~mL}$ of 1,2-dichlorobenzene was allowed to stand at $140{ }^{\circ} \mathrm{C}$ for $5 \mathrm{~h}$. The reaction mixture was chromatographed (silica gel, chlroform/ethyl acetate $2 / 1 \mathrm{v} / \mathrm{v})$ to obtain $21.6 \mathrm{mg}(63 \%)$ of Diels-Alder product 8 .

${ }^{1} \mathrm{H}$ NMR $\left(270 \mathrm{MHz}, \mathrm{CDCl}_{3}, 323 \mathrm{~K}\right): \delta 8.89$ (br s, 2H), 8.84 (s, 1H), 8.34 (s, 2H), 7.67 (br s, 4H), $7.42(\mathrm{~s}, 2 \mathrm{H}), 7.39(\mathrm{~s}, 2 \mathrm{H}), 7.36-7.28(\mathrm{~m}, 4 \mathrm{H}), 7.09(\mathrm{~s}, 2 \mathrm{H}), 7.06(\mathrm{~d}, J=8.1 \mathrm{~Hz}, 2 \mathrm{H}), 7.04$ $(\mathrm{d}, J=8.1 \mathrm{~Hz}, 2 \mathrm{H}), 6.80(\mathrm{~d}, J=8.4 \mathrm{~Hz}, 4 \mathrm{H}), 6.40(\mathrm{~d}, J=8.4 \mathrm{~Hz}, 4 \mathrm{H}), 4.40(\mathrm{~d}, J=3.9 \mathrm{~Hz}$, 4H), $4.09(\mathrm{t}, J=4.6 \mathrm{~Hz}, 4 \mathrm{H}), 3.82(\mathrm{~s}, 4 \mathrm{H}), 3.78(\mathrm{t}, J=4.6 \mathrm{~Hz}), 3.67(\mathrm{~s}, 4 \mathrm{H}), 3.63(\mathrm{~s}, 4 \mathrm{H}), 3.21$ (s, 4H), $2.96(\mathrm{~m}, 2 \mathrm{H}), 2.69-2.60(\mathrm{~m}, 4 \mathrm{H}), 1.30(\mathrm{~s}, 9 \mathrm{H}) \mathrm{ppm} .{ }^{13} \mathrm{C} \mathrm{NMR}\left(67.8 \mathrm{MHz}, \mathrm{CDCl}_{3}\right.$, 323K): $\delta 174.29,170.70,166.55,165.48,162.58,158.43,152.88,150.36,149.25,139.65$, 
$134.19,133.03,129.72,129.58,129.31,128.97,121.18,120.75,120.48,118.93,117.64$,

$117.61,116.63,112.69,112.40,100.47,70.95,69.72,67.46,60.42,52.15,44.79,41.19$,

35.17, 34.00, 31.13, 30.47, 21.15 ppm. IR (NaCl): 3354, 1737, 1644, 1600, 1534, 1262, 1161, $752 \mathrm{~cm}^{-1}$. MS (FAB, $\left.m \mathrm{NBA}\right): m / z(\%): 1455.6(100)\left[\mathrm{M}^{+}+\mathrm{H}\right]$.

\section{$\underline{\text { Tetraamide } 9}$}
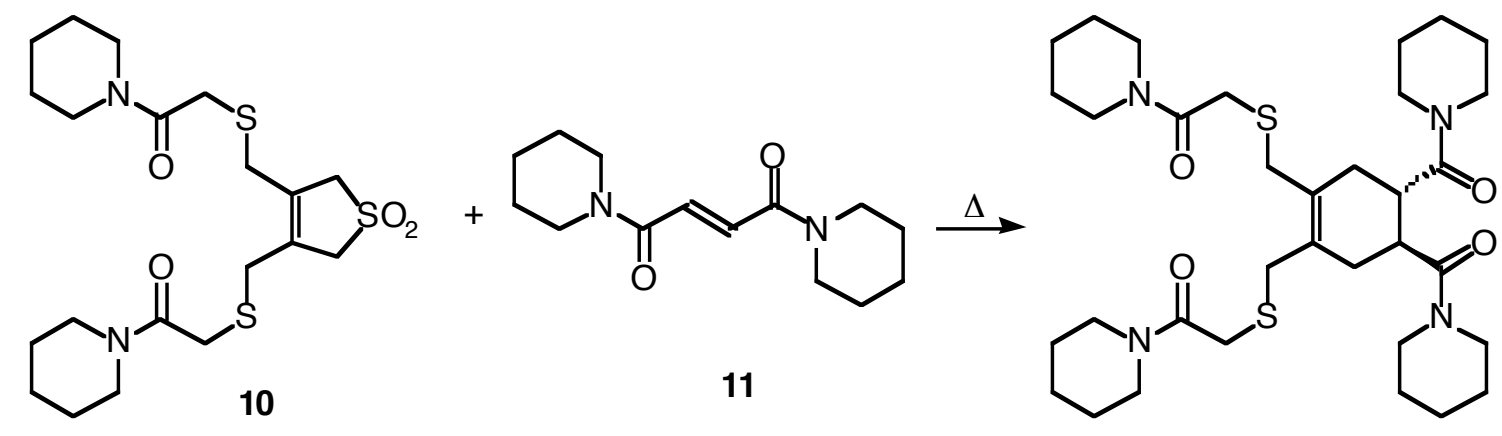

9

A solution of $151 \mathrm{mg}(0.326 \mathrm{mmol})$ of $\mathbf{1 0}$ and $82.1 \mathrm{mg}(0.326 \mathrm{mmol})$ of $\mathbf{1 1}$ in $0.3 \mathrm{~mL}$ of $1,2-$ dichlorobenzene was allowed to stand at $140{ }^{\circ} \mathrm{C}$ for $16 \mathrm{~h}$. The reaction mixture was diluted by chloroform, and directly purified by preparative GPC (chloroform) to give $140 \mathrm{mg}$ (66\%) of tetraamide 9 as a white crystal. This product was used as the standard material in the next experiment.

mp: 77.0-78.2 ${ }^{\circ} \mathrm{C} .{ }^{1} \mathrm{H}$ NMR (400 MHz, $\left.\mathrm{CDCl}_{3}, 296 \mathrm{~K}\right): \delta 3.54-3.48$ (m, 12H), 3.42-3.41 (m, 2H), $3.41(\mathrm{~s}, 4 \mathrm{H}), 3.29(\mathrm{t}, J=12.4 \mathrm{~Hz}, 8 \mathrm{H}), 2.42-2.31(\mathrm{~m}, 4 \mathrm{H}), 1.74-1.48(\mathrm{~m}, 24 \mathrm{H}) \mathrm{ppm} .{ }^{13} \mathrm{C}$ NMR (67.8 MHz, $\left.\mathrm{CDCl}_{3}, 323 \mathrm{~K}\right): \delta 172.69,167.08,129.24,47.46,46.87,42.90,38.89$, $34.22,33.69,32.33,26.66,26.38,25.68,25.53,24.71,24.37$ ppm. IR (NaCl): 3463, 2934, 2856, 1637, $1447 \mathrm{~cm}^{-1}$. MS (FAB, $\left.m \mathrm{NBA}\right): m / z(\%): 647.3(52)\left[\mathrm{M}^{+}+\mathrm{H}\right]$.

\section{Aminolysis of polymer 6}

A suspension of $7.8 \mathrm{mg}$ (5.67 $\mu$ mol-unit) of polymer 6 in $0.2 \mathrm{~mL}(2.0 \mathrm{mmol})$ of piperidine was allowed to stand at $40{ }^{\circ} \mathrm{C}$ for $48 \mathrm{~h}$, during which polymer gradually dissolved in piperadine. The yield of 9 was determined by quantitative HPLC analysis (ODS column, methanol/water $7 / 3 \mathrm{v} / \mathrm{v}$ ) of the crude product. 